\section{An admixture type of hyperplastic polyp at the esophagogastric junction}

A 67-year-old man with a 2-year history of reflux esophagitis visited our hospital complaining of heartburn. Physical examination was negative, and blood and biochemical laboratory tests were normal. Tumor markers were within the normal range. Upper gastrointestinal endoscopy identified a sessile polypoid lesion at the esophagogastric junction (EGJ) ( $\bullet$ Fig. 1). It was located just adjacent to the reddish scar of esophagitis ( Fig. 1). On chromoendoscopy using Lugol's solution, the protruding lesion was mostly iodine-unstained but iodine-stained in spots (๑ Fig. 2).
Following injection of saline into the submucosa, the polypoid lesion measuring $15 \mathrm{~mm}$ in size was successfully excised by endoscopic mucosal resection without complications. Microscopic examination showed that this lesion consisted of cardiac-type gastric mucosa with prominent foveolar hyperplasia ( $\bullet$ Fig. 3 ) and hyperplastic squamous epithelium ( $\bullet$ Fig.4). Thus, a diagnosis of admixture type of hyperplastic polyp of the EGJ was made. Hyperplastic polyp of the esophagus and EGJ are uncommon compared with that of the stomach. Abraham et al. studied 30 hyperplastic polyps of these sites from 27 affected patients and reported

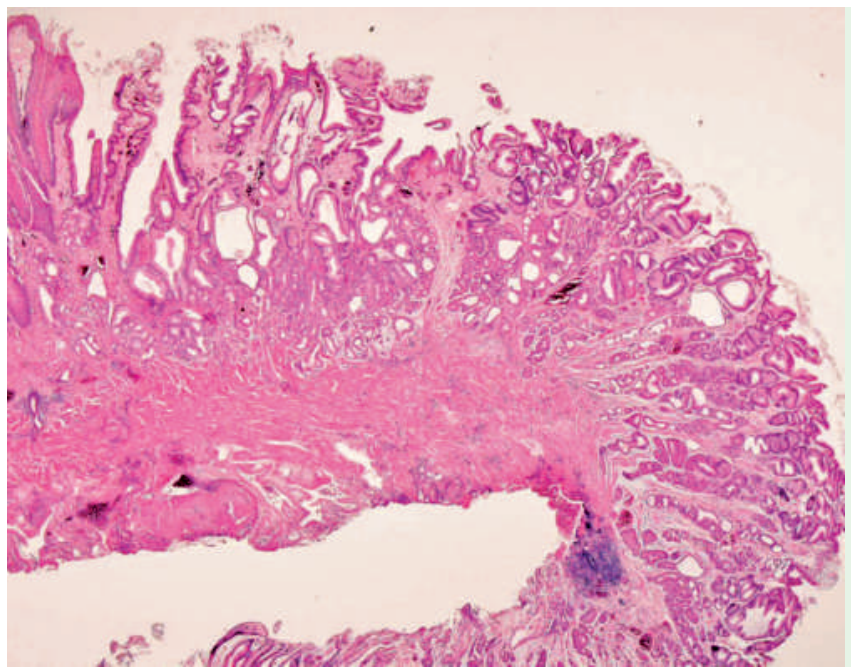

Fig. 3 Microscopic examination of the excised lesion by endoscopic mucosal resection showing hyperplastic proliferation of gastric foveolar epithelium.

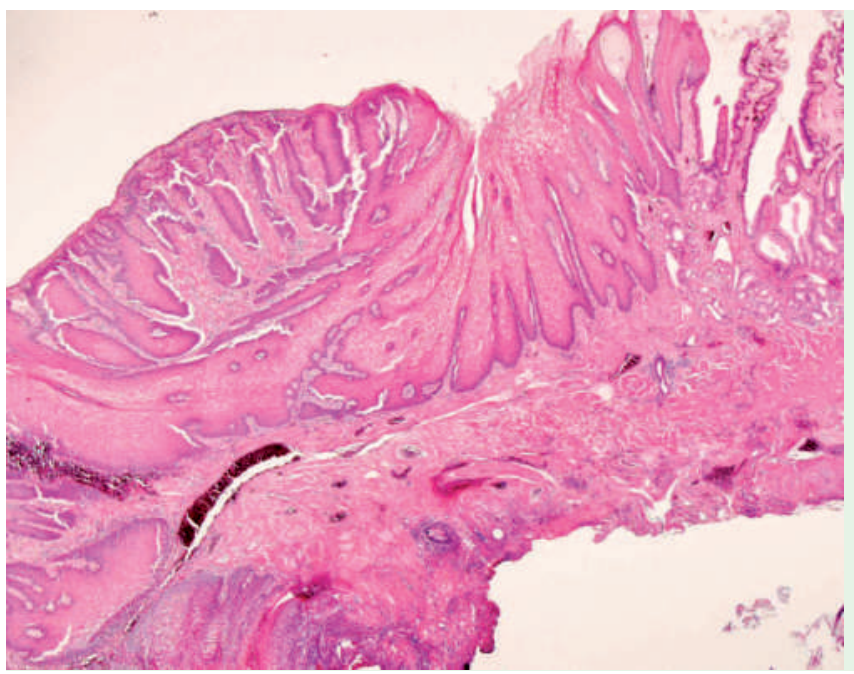

Fig. 4 Microscopic finding of hyperplastic proliferation of squamous epithelium.

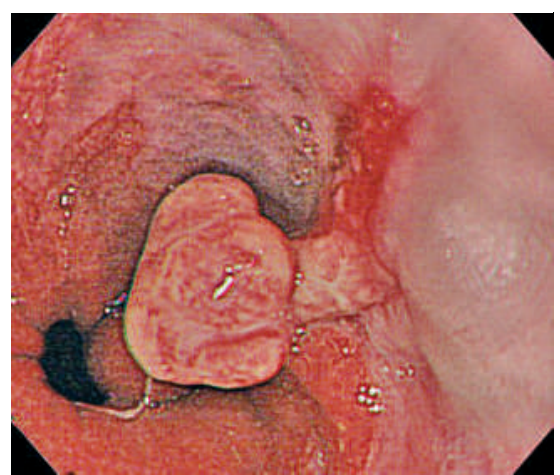

Fig. 1 Upper gastrointestinal endoscopy showing a sessile polypoid lesion at the esophagogastric junction, which was located near the reddish scar of reflux esophagitis.

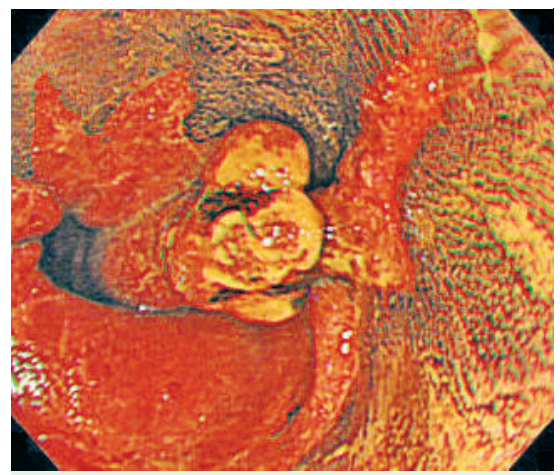

Fig. 2 Chromoendoscopy with Lugol's iodine solution showing the mottled stain pattern on the surface of polyp.

the clinicopathological characteristics. Most (80\%) were composed of predominantly cardiac mucosa, predominantly squamous mucosa (17\%), or rarely an admixture (3\%) [1]. Barrett's esophagus was present in only $15 \%$ of patients with the hyperplastic polyp, whereas there was an association between hyperplastic polyps of the esophagus/EGJ region and the presence of esophageal ulcer or erosive esophagitis, with the frequency being $67 \%$ in the affected patients [1], as seen in the present case. This may underscore the regenerative nature of hyperplastic polyps, which represent an exaggerated proliferation of foveolar or squamous epithelium, or both in areas of mucosal injury [1].

Endoscopy_UCTN_Code_CCL_1AB_2AC_3AB 
T. Hayashi ${ }^{1}$, S. Shikuwa ${ }^{1}$, H. Isomoto ${ }^{1}$, N. Yamaguchi ${ }^{1}$, K. Ohnita ${ }^{1}$, N. Inoue ${ }^{1}$, H. Nishiyama ${ }^{2}$, E. Fukuda ${ }^{2}$, Y. Mizuta ${ }^{1}$, S. Kohno

1 Second Department of Internal Medicine, Nagasaki University School of Medicine, Nagasaki, Japan

2 Department of Internal Medicine, National Nagasaki Medical Center, Nagasaki, Japan

\section{References}

1 Abraham SC, Singh VK, Yardley JH et al. Hyperplastic polyps of the esophagus and esophagogastric junction. Am J Surg Pathol 2001; 25: $1180-1087$

\section{Bibliography}

DOI $10.1055 / \mathrm{s}-2007-995488$

Endoscopy 2008; 40: E66-E67

(c) Georg Thieme Verlag KG Stuttgart · New York . ISSN 0013-726X
Corresponding author

\section{H. Isomoto, MD}

Second Department of Internal Medicine Nagasaki University School of Medicine 1-7-1 Sakamoto

Nagasaki, 852-8501

Japan

Fax: + 81-95-8497285

hajimei2002@yahoo.co.jp 